\title{
Nuclear data activities at the NEA Data Bank
}

\author{
A. Hasegawa, H. Henriksson, F.J. Mompeán, C. Nordborg, Y. Rugama, and E. Sartori \\ OECD Nuclear Energy Agency, 12 boulevard des Îles, 92130 Issy-les-Moulineaux, France
}

\begin{abstract}
The OECD Nuclear Energy Agency (NEA) Data Bank is an international centre of reference for its member countries with respect to basic nuclear tools, such as computer codes and nuclear data. The Data Bank is part of an international network of data centres in charge of the compilation and dissemination of basic nuclear data. The NEA nuclear data services include the collection of data, validation and distribution of the Nuclear Data libraries via the NEA website, offering easy access to databases containing bibliographical and experimental information, as well as evaluated libraries, e.g., the Joint Evaluated Fission and Fusion (JEFF) library. The selection and distribution of chemical thermodynamic data for radioactive waste management applications is the subject of the Thermochemical Database Project (TDB), supported by 17 organisations in 12 member countries and co-ordinated by the NEA Data Bank. In support of data evaluation, and generation of project oriented data libraries, relevant computer codes in the field of nuclear models, experimental data processing and evaluated data processing are made available to experts with the support of the member countries. Large collections of benchmark experiments for data and code validation are also available from the NEA in areas such as criticality safety (ICSBEP), radiation shielding (SINBAD), fuel performance (IFPE) and reactor physics (IRPhE). The NEA Working Party on international nuclear data Evaluation Co-operation (WPEC) provides a framework for co-operative activities between the six major evaluation projects (BROND, CENDL, ENDF, FENDL, JEFF and JENDL).
\end{abstract}

\section{Introduction}

The Nuclear Energy Agency (NEA) is a specialised agency within the Organisation for Economic Co-operation and Development (OECD). The mission of the NEA is to assist its Member countries in maintaining and further developing, through international co-operation, the scientific, technological and legal bases required for the safe, environmentally friendly and economical use of nuclear energy for peaceful purposes.

The Data Bank is a part of the NEA, but has its own membership and a separate budget. Its primary role is to provide scientists in member countries with reliable nuclear data and computer programs for use in different nuclear applications. The services include also chemical thermodynamic data for radioactive waste management applications. The 22 member countries of the Data Bank are Austria, Belgium, Czech Republic, Denmark, Finland, France, Germany, Greece, Hungary, Italy, Japan, Republic of Korea, Mexico, Netherlands, Norway, Portugal, Slovak Republic, Spain, Sweden, Switzerland, Turkey, and United Kingdom. Users of the Data Bank services include governmental research institutes, industry and universities.

The NEA Data Bank organises seminars and workshops to present information on computer programs or groups of programs that are considered to be of special interest to users. Training courses on widely-used computer programs are organised a few times a year to ensure a correct and effective use of them. An example is the NJOY user's group meeting that was organised in connection with the biannual JEFF working group meeting in November 2006. All information can easily be found on the NEA Data Bank web page (see fig. 1).

\section{International collaboration}

The NEA Data Bank is involved in many international collaborations, such as the network of nuclear reaction data centres (NRDC). The NRDC was established in the 1960s to collect and disseminate experimental data, addressing for example the data needs of the fission reactor industry. The present aim of the NRDC is to encompass all types of nuclear reaction data, including neutron- and charged-particle-induced data, as well as photonuclear data, considering the requirements of many other nuclear applications, such as accelerator driven systems, fusion reactors, nuclear medicine, materials analysis, environmental monitoring, and basic research [1].

\section{Nuclear data}

The NEA nuclear data services include the collection of data, validation and distribution of evaluated nuclear data libraries via the NEA website. Our on-line databases provide a free access to nuclear data in the following categories:

- EVA: Evaluated nuclear data libraries (JEFF-3.1, ENDF/B-VII.0, JENDL-3.3 and many more) in a common format describing reaction data, decay data, fission yields, etc.

- EXFOR: Experimental nuclear reaction data, covering neutron and charged particle induced reactions, as well as photonuclear data.

- CINDA: Bibliographical information on experimental, theoretical and evaluated nuclear data.

The EVA Web retrieval page is shown in figure 2. Links to the EXFOR and CINDA retrieval pages are easily available from there, as well as links to relevant computer codes for nuclear data. 


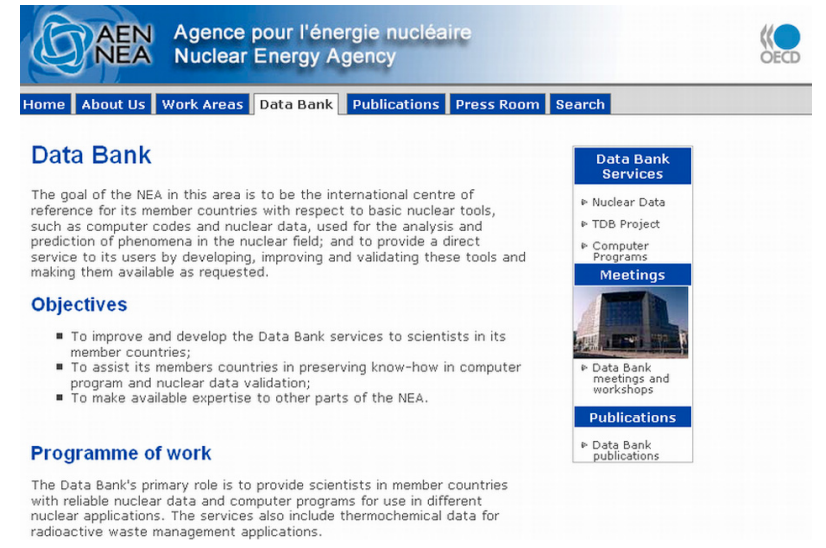

Fig. 1. NEA Data Bank home page, www.nea.fr/html/databank/, where information about nuclear data, computer programs, chemical themodynamic data, and upcoming meetings are available.

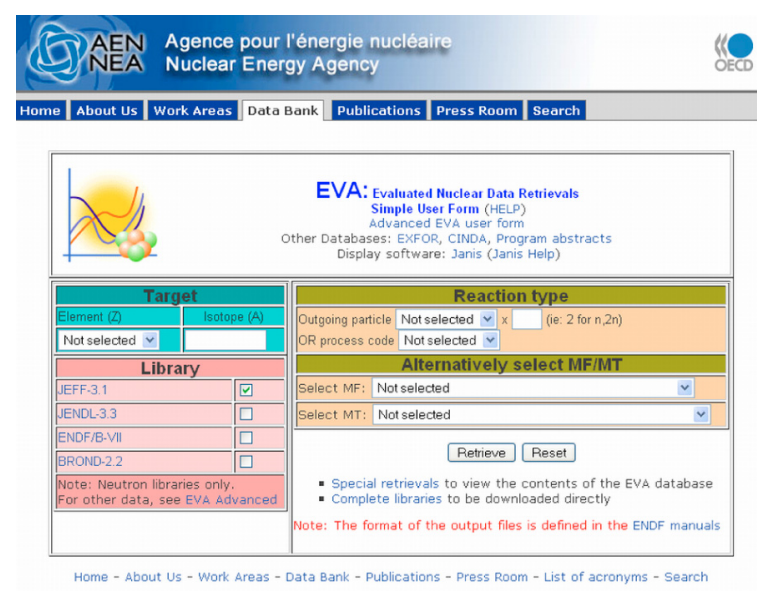

Fig. 2. The Web retrieval system EVA for evaluated data (www.nea.fr/html/dbdata/eva/). The search pages for EXFOR and CINDA are easily available from this Web page.

\subsection{Experimental data}

A comprehensive set of experimental reaction data are stored in a database called EXFOR, that was initiated already in 1969 with the merge of several databases into a standardised format. EXFOR has always been coordinated through the international NRDC network [1] where the three main nuclear data centres, besides the OECD/NEA Data Bank, are: the National Nuclear Data Center (NNDC) at Brookhaven National Laboratory (USA), the Nuclear Data Services (NDS) at IAEA and the Nuclear Data Center (CJD) at the Institute of Physics and Power Engineering (IPPE) in Russia.

In addition to storing the experimental data points and their bibliographic information, information including source of uncertainties is also compiled. EXFOR is complete with respect to neutron reaction data, and is intended to cover also all charged particle data up to ${ }^{12} \mathrm{C}$ as incident particle and with incident energies up to about $1 \mathrm{GeV}$. Selected photon-induced and exotic reaction data are also included. EXFOR contains at present about 16,000 experiments divided in 124,000 different data sets.
The bibliographic database CINDA is closely linked to EXFOR, and contains an almost complete bibliography of all neutron data published since 1932, as well as an index to corresponding EXFOR entries and evaluated data. Besides neutron data, CINDA also covers charge-particle induced data, photo-neutron, photo-fission and spontaneous fission data. CINDA is available on web retrieval, through the JANIS program, and as an archive book that can be requested from the NEA [2].

\subsection{Evaluated data}

The Joint Evaluated Fission and Fusion (JEFF) project is a collaboration between the countries participating in the NEA Data Bank. The JEFF library comprises sets of evaluated nuclear data, mainly for fission and fusion applications; it contains a number of different data types, including neutron and proton interaction data, radioactive decay data, fission yield data, thermal scattering law data and photo-atomic interaction data [3].

The JEFF project also encompasses the European Fusion File (EFF) and European Activation File (EAF) projects (funded by the EC Fusion Programme). These projects are directed by the EFF/EAF monitor group, who holds meetings in conjunction with the other JEFF working groups. Joint sessions are organised to discuss matters of common interest for all the JEFF working groups.

The JEFF-3.1 Nuclear Data Library is the latest version and it was released in May 2005. It contains data evaluations collected and checked at the NEA Data Bank in cooperation with several member country laboratories. Within the framework of the JEFF project, the JEFF Working Group on Radioactive Data and Fission Yields decided to produce improved versions of the decay data and fission-yield libraries with a release in conjunction with the JEFF library. Activation data has also been included in the latest version, based on the EAF-2003 library.

The JEFF-3.1 neutron data library covers 381 isotopes, which is an increase from 340 in JEFF-3.0, the preceding library released in 2002. There are 26 isotopes in the proton data library, and 9 materials are covered in the thermal scattering law file. A great achievement was to include covariance data for many isotopes in the neutron data library. The special purpose library on activation data contains 774 target nuclei with over 12,600 neutron induced reactions. Included is also radioactive decay data with 3,852 isotopes and spontaneous and neutron induced fission yield data. The contents of the JEFF-3.1 library is visualised in figure 3, where all available nuclides in the neutron- and radioactive decay data libraries are shown.

The full documentation of JEFF-3.1, JEFF Report 21, was published 2006, and is available from the NEA [4].

Processed data libraries in the ACE format for MCNP based on JEFF-3.1 have been prepared for several temperatures [5]. The library can be requested from the Computer Program Services.

The NEA Working Party on international nuclear data Evaluation Co-operation (WPEC) provides a framework for collaboration between the six major evaluation projects 


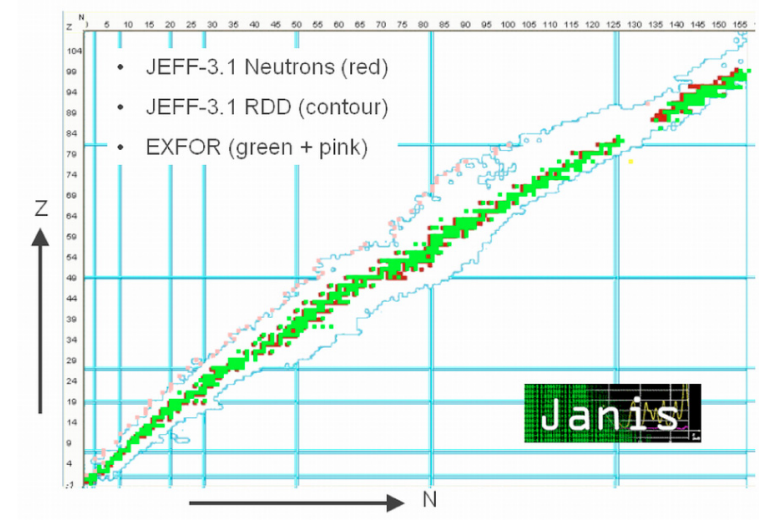

Fig. 3. Chart of nuclides of the JEFF neutron library, the radioactive decay data (RDD) library, and EXFOR.

(BROND, CENDL, ENDF, FENDL, JEFF and JENDL) [7]. Several subgroups of the WPEC are at present working of future needs for evaluations and uncertainties of data, such as the high priority nuclear data request list (HPRL). The most recent WPEC subgroups are

- Covariance Data in the Fast Neutron Region,

- Assessment of Fission Product Decay Data for Decay Heat Calculations,

- Nuclear Data Needs for Advanced Reactor Systems,

- Prompt Photon Production from Fission Products,

- Processing of Covariance Data.

\subsection{Request list of nuclear data}

The HPRL is a collection of experimental data requests needed for new evaluations to be used in nuclear industry and for future nuclear applications (e.g., Generation-IV reactors or ITER) [8]. The HPRL therefore provides a guide for those planning new measurements, nuclear calculations and evaluation programmes.

Requests are reviewed by external referees and are divided into high priority requests, where a quantitative justification is needed, and general requests, where a more qualitative justification is sufficient. There are at the moment nine requests available for consideration that have been reviewed.

The HPRL project is organised through the WPEC and the request list is maintained by the NEA Data Bank. The HPRL is presented on the web page: www.nea.fr/html/dbdata/hprl/.

\subsection{The display program JANIS}

A computer program, JANIS, to display, compare and manipulate data is developed by the NEA Data Bank. JANIS has recently been upgraded, and the new version (JANIS3.0) is being tested at present, with a planned release in May 2007 [9]. JANIS is designed to allow the user access to numerical and graphical representations without prior knowledge of the data format.

JANIS comprises a number of functionalities and contains the most recent evaluated data libraries, e.g. JEFF3.1, JENDL-3.3, and ENDF/B-VII.0. The search function for experimental data information in EXFOR and CINDA has been modified, and the EXFOR search window gives now the user full access to the database. A variety of output formats exist in JANIS. For the graphical display, the PS/EPS and PNG formats are possible, and tabular data can be stored in CSV format for further use in other software (e.g., MS Excel). Updates of the software can be automatically downloaded through the liveupdate feature. Feedback is appreciated and can be posted at Janisinfo@nea.fr.

\section{Chemical thermodynamic data}

The selection and distribution of chemical thermodynamic data for radioactive waste management applications is the subject of the Thermochemical Database Project (TDB), supported by 17 organisations in 12 member countries and coordinated by the NEA Data Bank [10].

The TDB project examines the key elements required for geochemical modelling of underground repositories. Teams of international experts carry out critical reviews of bibliographic references and propose a quality-assured database. Detailed reports are published in the open literature and the database is accessible free of charge to OECD member countries (see: www.nea.fr/html/dbtdb).

Recent reports on Nickel (2005), Selenium (2005), Zirconium (2005) and Organic Ligands (2005) have been published and current work is on Thorium, Tin, Iron and the Thermodynamics of Solid Solutions. A new phase of the project is planned for 2008-2011 with emphasis in new reviews and updates of the existing database.

\section{Computer programs and benchmarks}

In support of data evaluation, and generation of project oriented data libraries, relevant computer codes in the field of nuclear models, experimental and evaluated data processing are made available to member countries. This computer program service includes collection of programs, compilation and verification of the completeness of the computer program package and its documentation. The computer program collection covers a wide range of nuclear energy and radiation physics applications, including codes for preparation of application data, such as NJOY for the processing of evaluated data into the ACE format used by MCNP.

The Data Bank collaborates with the Radiation Safety Information Computational Center (RSICC) [11] in the US and the IAEA concerning the distribution of programs and processed data.

International computer code comparisons and integral benchmark experiment calculations are carried out in cooperation with the Nuclear Science Committee of the NEA. Results from international benchmark comparisons are incorporated in the documentation of the computer programs and nuclear data distributed by the Data Bank to users on request. These collections of benchmark experiments for data and code validation are available in areas such as criticality safety (ICSBEP), fuel performance (IFPE), radiation shielding (SINBAD) and reactor physics (IRPhE). 
The International Handbook of Evaluated Criticality Safety Benchmark Experiments (ICSBEP) have increased from 379 to 442 evaluations, containing benchmark specifications for 3,955 critical or sub-critical configurations, 23 criticality-alarm-placement/shielding configurations with multiple dose points for each, and 20 configurations that have been categorized as fundamental physics measurements relevant to criticality safety applications (fission rate data for ${ }^{239} \mathrm{Pu},{ }^{235} \mathrm{U},{ }^{238} \mathrm{U}$, and ${ }^{237} \mathrm{~Np}$ ) in the 2006 edition of the ICSBEP Handbook.

The international database SINBAD is an established set of radiation shielding and dosimetry data [13]. Over 80 experiments are at present available in SINBAD, relevant in reactor and accelerator shielding, as well as in fusion blanket neutronics. In addition to the experimental results together with uncertainties, the database includes characterization of the radiation source, description of the shielding materials and the relevant detectors used, as well as the transport computer code inputs used for the interpretation of the experiment.

\section{Recent publications}

The NEA publishes many studies related to the application of nuclear data. Below follows a list of some recent publications from the NEA Data Bank on nuclear data relevant issues, mostly free of charge, on request from the NEA:

- Perspectives on Nuclear Data for the Next Decade Workshop Proceedings, Bruyères-le-Châtel, France, 26-28 September 2005 (2006).

- Chemical Thermodynamics of Organic Ligands (Vol. 9) (2005), on sale.

- The JEFF-3.1 Nuclear Data Library, JEFF Report 21 (2006).

- CINDA Archive 2006 The Comprehensive Index to Literature and Computer Files on Nuclear Reaction Data (2007, to be published).

The following publications were published within the framework of the WPEC activities and under the Nuclear Science Committee of the NEA:

- Computer Simulation of MASURCA Critical and Subcritical Experiments MUSE-4 Benchmark - Final Report (2006).

- International Evaluation Co-operation (Vol. 19) Neutron Activation Cross-section Measurements from Threshold to $20 \mathrm{MeV}$ for the Validation of Nuclear Models and Their Parameters (2005).
- International Evaluation Co-operation (Vol. 20) Covariance Matrix Evaluation and Processing in the Resolved/Unresolved Resonance Regions (2006).

- International Evaluation Co-operation (Vol. 21) Assessment of Neutron Cross-section Evaluations for the Bulk of Fission Products (Vol. 21) (2005).

- International Evaluation Co-operation (Vol. 22) Nuclear Data for Improved LEU-LWR Reactivity Predictions (Vol. 22) (2006).

\section{References}

1. H. Henriksson et al., The art of collecting experimental data internationally: EXFOR, CINDA and the NRDC network (these proceedings).

2. CINDA Archive 2006, The comprehensive Index to Nuclear Reaction Data (1935-2006), OECD Nuclear Energy Agency (2007, to be published).

3. A. Koning et al., The JEFF evaluated nuclear data project (these proceedings).

4. JEFF Report 21, The JEFF-3.1 Nuclear Data Library, NEA (2006), ISBN 92-64-02314-3.

5. O. Cabellos, Y. Rugama, Processing and validation of JEFF3.1 library in ACE format at 10 different temperatures (these proceedings).

6. W. Haeck, B Verboomen, et al., Aleph-DLP 1.1.0. Creation cross-section libraries for MCNP(X), SCK, CEN-BLG-1002, Rev. 0, 2006.

7. A. Koning et al., Status and Future Work of the NEA Working Party on International Nuclear Data Evaluation Cooperation (these proceedings).

8. A. Plompen et al., The NEA High Priority Nuclear Data Request List for future needs (these proceedings).

9. N. Soppera et al., Recent upgrades of the Data display tool JANIS (these proceedings).

10. F.J. Mompean, H. Wanner, Radiochim. Acta 91, 617 (2003).

11. B.L. Kirk, H.T. Hunter, The Radiation Safety Information Computational Center (RSICC) - Forty Years of Nuclear Knowledge Management, in International Conference on Nuclear Knowledge Management, 7-10 Sept. 2004, Saclay, France, IAEA-CN123/03/P/17.

12. J.B. Briggs et al., Integral benchmark data for nuclear data testing through the ICSBEP and the newly organized IRPHEP (these proceedings).

13. I. Kodeli, E. Sartori, B. Kirk, SINBAD Shielding Benchmark Experiments Status and Planned Activities, in ANS 14th Top. Meeting of Rad. Prot. and Shielding Division, Carlsbad, USA, 2006, and SINBAD database: http://www.nea.fr/html/science/shielding/. 